\title{
SUNCT and trigeminal neuralgia attributed to meningoencephalitis
}

\author{
Pablo Eguia · Juan Carlos Garcia-Monco • \\ Nuria Ruiz-Lavilla - Vanesa Diaz-Konrad · \\ Fernando Monton
}

Received: 5 November 2007 / Accepted: 7 December 2007/Published online: 23 January 2008

(c) Springer-Verlag 2008

\begin{abstract}
We describe a 46-year-old female with viral meningoencephalitis (likely varicella-zoster virus) who developed a SUNCT syndrome followed a few days later by trigeminal neuralgia. Both disorders resolved in parallel with the resolution of encephalitis, which suggests a causal link. In conclusion, headache attributed to intracranial infection may have the clinical features of SUNCT or TN.
\end{abstract}

Keywords SUNCT TACs · Trigeminal neuralgia · Meningoencephalitis · VVZ · Varicella-zoster

\section{Introduction}

The IHS classification of headache disorders (ICHD-II) [1] establishes that a headache occurring for the first time in close temporal relation to an infection should be coded as a secondary headache attributed to the infection.

Short-lasting unilateral neuralgiform headache attacks with conjunctival injection and tearing syndrome (SUNCT) and trigeminal neuralgia (TN) are occasionally secondary to space-occupying lesions in the posterior fossa or involving the pituitary gland, but its association to viral encephalitis has not been reported so far.

We describe a patient with SUNCT syndrome and coexistent TN occurring over the course of an acute

P. Eguia $(\bowtie) \cdot$ N. Ruiz-Lavilla · V. Diaz-Konrad · F. Monton Department of Neurology, Hospital Nuestra Señora de Candelaria, Carretera del Rosario 145,

38010 Santa Cruz de Tenerife, Spain

e-mail: peguia@telefonica.net

J. C. Garcia-Monco

Department of Neurology, Hospital de Galdacano,

Vizcaya, Spain meningoencephalitis probably due to varicella-zoster virus $(\mathrm{VZV})$ reactivation.

\section{Case report}

A 46-year-old woman presented with 1 week of fever. On the day of admission she developed somnolence and headache. She described right-sided episodes of severe retro-orbital burning pain, lasting 30-60 s, associated with prominent lacrimation, conjunctival injection, ptosis and rhinorrhea. She had up to 10 attacks per hour. No triggers were identified. Her past medical history revealed episodic migraine without aura and smoking.

On examination, she was somnolent, had mild neck stiffness and fever of $39^{\circ} \mathrm{C}$. Cranial nerve examination, including the corneal reflex, was normal. Serum chemistry values, CBC and differential were normal. A brain CT scan without contrast was normal. Lumbar puncture revealed 40 white cells $/ \mathrm{mm}^{3}$ (88\% monocytes), 20 red cells $/ \mathrm{mm}^{3}$, protein of $42 \mathrm{mg} / \mathrm{dL}$, and glucose of $70 \mathrm{mg} / \mathrm{dL}$. CSF Gram stain was unrevealing and culture came negative. Intravenous acyclovir was started for presumed herpes simplex meningoencephalitis, and was administered for 10 days. Also, a diagnosis of SUNCT syndrome was made, and gabapentine and morphine were initiated. The following day, EEG demonstrated diffuse slowing without epileptic discharges.

On day 2 she developed an additional, different type of facial pain, consisting of severe sharp, electric shock-like attacks, less than $5 \mathrm{~s}$ in duration, over the second division of the right trigeminal nerve, without associated autonomic features. Attacks occurred spontaneously several times a day but were also triggered by eating and touching the nose or the upper lip. A diagnosis of TN was made. 
On day 3, MRI revealed multiple lesions in both cerebral hemispheres located at the cortical-white matter junction with increased signal intensity on T2-weighted sequences (Fig. 1). T1-gadolinium sequences demonstrated mild lesion enhancement.

On day 5 she developed partial motor seizures and valproic acid (1,500 mg daily) was substituted for gabapentine. Progressively, fever disappeared, her mental status recovered to normal, and there was a gradual decrease in the frequency and intensity of both types of pain over the following days. IgM and IgG against VZV were positive in serum. VZV serology and PCR in CSF were not performed. Herpes simplex virus antibodies were negative in serum and CSF; Herpes simplex PCR was not performed. Other specific studies for viral, fungal and bacterial agents including HIV were negative.

Two weeks after admission, TN had completely resolved, and she was discharged on valproic acid. One month after admission SUNCT attacks remained at a lower frequency (3-4/day); they were triggered by bright light. Two months later, SUNCT attacks had resolved except for the dysautonomic features. She continued to experience 3 or 4 daily episodes, of lacrimation with conjunctival injection and rhinorrhea that occurred at the same time of the day, particularly upon morning awakening. The episodes were painless, and rather, she described a right eye discomfort, like a foreign body. They occurred with a highly variable frequency, between 1 and 4 days a week and 1-3 times a day, and lasted up to $15 \mathrm{~min}$. An ophthalmological evaluation was unremarkable. Valproic acid was tapered off and stopped. Six months later the autonomic signs and symptoms completely resolved. The episodic ocular discomfort remained, and was still present 15 months later.

\section{Discussion}

This report describes the unusual association of SUNCT syndrome with TN over the course of a viral meningoencephalitis. The temporal relationship between the onset and resolution of meningoencephalitis and pain attacks suggests that these events were linked. Furthermore, the ICHD-II [1] indicates that a new headache in close temporal relation to an infection should be coded as a secondary headache attributed to the infection. Although pain characteristics may mimic those of primary headaches, headaches associated with infection are not well characterized, and this chapter of the ICHD-II remains controversial [2]. It is of interest that this patient's past medical history revealed episodic migraine without aura, but the infection did not elicit migraine attacks.

The association of TN with SUNCT has been reported before. Bouhassira et al. [3] and Sesso [4] described patients with SUNCT syndrome evolving from V1 TN, raising the question of whether these two entities are pathogenetically distinct conditions or ends of the same spectrum.

Leone et al. [5] suggested that a disturbance of hypothalamic neurotransmitter regulation might result in variable activation patterns of the trigeminal system, explaining some of the different clinical characteristics of the short-lasting unilateral headaches, such as their varying duration and accompanying autonomic symptoms. It could be argued that this patient had secondary forms of headache mimicking the primary syndromes due to the encephalitic injury of structures involved in the pathogenesis of these syndromes, perhaps on a background of individual susceptibility. Alternatively, viral infection could have resulted in irritation/inflammation of the
Fig. 1 Axial fluid-attenuated inversion recovery image (a) shows two hyperintense lesions (arrows) located at the corticalwhite matter junction. Axial T1gadolinium sequence (b) demonstrates mild enhancement (arrow) of an occipital lobe lesion
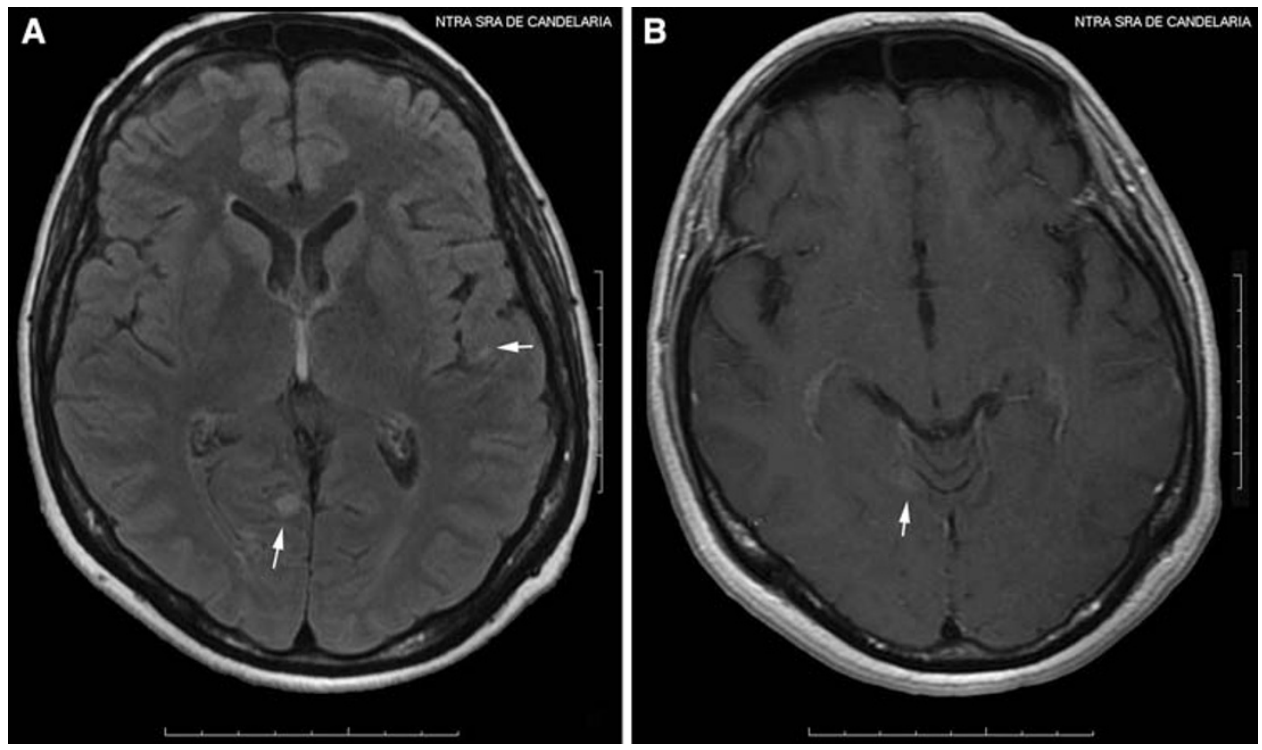
trigeminal root, thus triggering pain attacks. It should be noted that this patient did not develop any facial herpetic rash, and that pain characteristics were atypical for an herpetic neuralgia.

The persistence of circadian dysautonomic features in the absence of pain for several weeks in our patient suggests a hypothalamic abnormality with subsequent trigemino-vascular and cranial autonomic activation, and is consistent with the proposed pathophysiology of SUNCT [6]. This dissociation between pain and autonomic features has been reported before in one patient with SUNCT [6], and in patients with cluster headache $(\mathrm{CH})$ [7].

The residual feeling of a foreign body in the eye with normal ophthalmological evaluation is also interesting. Some patients with hemicrania continua (HC) describe a sensation of ocular discomfort, similar to that of a foreign body or "sand" in the eye [8], and in a series of 43 SUNCT patients one of them referred an ipsilateral gritty eye [6]. HC and SUNCT share posterior hypothalamus activation and dysautonomic features [9]. Hence, residual "foreign body in the eye" feeling could reflect a dysfunction of the trigeminal-autonomic system.

Meningoencephalitis in this patient was likely caused by $\mathrm{VZV}$, as the serology suggested, and is usually the result of viral reactivation $[10,11]$. VZV meningoencephalitis without an accompanying rash, like in our patient, has been described before [12,13], but we have only found two case reports on the association of acute onset TN or TACs with varicella-zoster. A physician reported his own case of acute-onset TN associated with a significant rising in VZV antibodies [14]. Giacovazzo et al. [15] reported a case of chronic paroxysmal hemicrania $(\mathrm{CPH})$ related to an ophthalmic herpes-zoster infection.

SUNCT syndrome is often refractory to medical therapy and Lamotrigine may be considered the first choice [16]. Therapy in this patient, however, was influenced by the underlying process and the presence of motor seizures. Valproic acid is not a first-line drug for SUNCT or TN. Thus, it is likely that the headaches ceased spontaneously after meningoencephalitis resolved, which again supports a causal link.

In summary, headache attributed to encephalitis may mimic primary syndromes like SUNCT and TN. The hypothetical role of neurotropic viruses, such as VZV, in the pathogenesis of TACs remains to be clarified.

Acknowledgments The authors are grateful to Dr. Vicente Martin, from the Radiology Department of our institution, for providing us with the MR images.
Conflicts of interest None.

\section{References}

1. Headache Classification Committee of the International Headache Society (2004) The international Classification of headache Disorders, 2nd edn. Cephalalgia 2004; 24(Suppl 1):1-160

2. Marchioni E, Tavazzi E, Bono G, Minoli L, Bastianello S, Sinforiani E, Sances G, Tinelli C, Nappi G (2006) Headache attributed to infection: observations on the IHS classification (ICHD-II). Cephalalgia 26:1427-1433

3. Bouhassira D, Attal N, Esteve M, Chauvin M (1994) SUNCT syndrome. A case of transformation from trigeminal neuralgia? Cephalalgia 14:168-170

4. Sesso RM (2001) SUNCT syndrome or trigeminal neuralgia with lacrimation and conjunctival injection? Cephalalgia 21:151-153

5. Leone M, Mea E, Genco S, Bussone G (2006) Coexistence of TACS and trigeminal neuralgia: Pathophysilogical conjectures. Headache 46:1565-1570

6. Cohen AS, Matharu MS, Goadsby PJ (2006) Short-lasting unilateral neuralgiform headache attacks with conjunctival injection and tearing (SUNCT) or cranial autonomic features (SUNA)-a prospective clinical study of SUNCT and SUNA. Brain 129:2746-2760

7. Lin H, Dodick DW (2005) Tearing without pain after trigeminal root section for cluster headache. Neurology 65:1650-1651

8. Newman LC, Lipton RB, Solomon S (1994) Hemicrania continua: ten new cases and a review of the literature. Neurology 44:2111-2114

9. Goadsby PJ, Lipton RB (1997) A review of paroxysmal hemicranias, SUNCT syndrome and other short-lasting headaches with autonomic feature, including new cases. Brain 120:193-209

10. Gilden DH, Kleinschmidt-DeMasters BK, LaGuardia JJ, Mahalingam R, Cohrs RJ (2000) Neurologic complications of the reactivation of varicella-zoster virus. N Engl J Med 342(9):635645

11. Gilden DH, Dueland AN, Devlin ME, Mahalingam R, Cohrs RJ (1992) Varicella-zoster reactivation without rash. J Infect Dis 166(Suppl 1):S30-S34

12. Echevarria JM, Casas I, Tenorio A, de Ory F, Martinez-Martin P (1994) Detection of varicella-zoster virus-specific DNA sequences in cerebrospinal fluid from patients with acute aseptic meningitis and no cutaneous lesions. J Med Virol 43:331-335

13. Powell KF, Wilson HG, Croxson MO, Marshall MR, Wong EH, Anderson NE, Thomas MG (1995) Herpes zoster meningoencephalitis without rash: Varicella zoster virus DNA in CSF. J Neurol Neurosurg Psychiatry 59(2):198-199

14. Easton HG (1970) Zoster sine herpete causing acute trigeminal neuralgia. Lancet 2:1065-1066

15. Giacovazzo M, Di Sabato F, Gallo MF, Granata M, Martelletti P (1992) Chronic paroxysmal hemicrania following ophthalmic herpes zoster. Riv Eur Sci Med Farmacol 14(1):45-47

16. Cohen AS, Matharu MS, Goadsby PJ (2007) Trigeminal autonomic cephalalgias: current and future treatments. Headache 47(6):969-980 